\title{
Neurological health and premature ovarian insufficiency - pathogenesis and clinical management
}

\author{
Radosław Słopień \\ Department of Gynaecological Endocrinology, Poznan University of Medical Sciences, Poznan, Poland
}

\begin{abstract}
Premature ovarian insufficiency $(\mathrm{POI})$ is related to neurological problems through neurological symptoms of oestrogen deficiency, diseases caused by oestrogen deficiency, and neurological genetic diseases. Neurological symptoms of oestrogen deficiency are usually reported as climacteric symptoms. Diseases caused by oestrogen deficiency are dementia, cognitive decline, and Parkinsonism. Among genetic neurological disorders, ovarioleukodystrophy and fragile X-associated tremor/ataxia syndrome (FXTAS) are reported.
\end{abstract}

Key words: premature ovarian insufficiency, climacteric symptoms, dementia, Parkinsonism.

\section{Introduction}

Neurological problems of women with untreated premature ovarian insufficiency (POI) are usually related to the three following areas: symptoms of oestrogen deficiency, diseases related to oestrogen deficiency, and genetic diseases including POI as a one of the clinically relevant features.

\section{Symptoms of oestrogen deficiency}

Oestrogen deficiency is a cause of climacteric symptoms, which are usually subdivided into five categories as follows: menstrual bleeding disorders; vegetative symptoms; psychosomatic symptoms; somatotrophic changes; and metabolic changes [1]. Among vegetative symptoms: hot flushes, night sweating, insomnia, vertigo, arthralgia, headaches, palpitations, and paraesthesia and among psychosomatic symptoms: nervousness, low mood, and general weakness are reported.

Gibson-Helm et al. [2] reported that climacteric symptoms like sleeping difficulties, loss of interest in most things, feeling unhappy or depressed, crying spells, irritability, hot flushes, sweating at night, and loss of interest in sex were more frequent and more severe in women with premature ovarian insufficiency (POI) compared to premenopausal controls. Only one symptom: feeling tired/lacking in energy was less frequent in $\mathrm{POI}$ women than in premenopausal controls (43\%). The same authors reported differences between the symptoms in women with medically-induced POI and idiopathic POI. Women with medically-induced
POI reported the following more frequently: sleeping difficulties (69\%) and feeling tired/lacking in energy (69\%), whereas women with idiopathic POI reported more frequently loss of interest in sex (52\%).

\section{Diseases related to oestrogen deficiency}

\section{Dementia and cognitive decline}

Both laboratory research and clinical studies suggest that there is an association between POI and an increased risk of developing dementia and cognitive decline [3].

Laboratory studies revealed that oestrogen improves synaptic plasticity acting on the level of dendritic spines in hippocampi of oophorectomised rats, it improves cerebral blood flow and glucose metabolism, and has an antioxidative effect on the neuronal level. It has also been reported to increase choline acetyltransferase activity in the basal forebrains and hippocampi of oophorectomised rats, reduce the deposition of amyloid in the brain, and prevent amyloid from inducing a rise in intracellular calcium and from causing mitochondrial damage [4]. Oestrogen acts in neurons and glial cells via the intracellular oestrogen receptors $\alpha$ and $\beta[5,6]$ as well as antiatherogenic actions including enhancement of endothelial function, blockage of smooth muscle cell proliferation, and inhibition of inflammation [7].

Most clinical studies have shown a negative effect of surgical menopause on memory and cognitive functions. Such data were reported in the Mayo Clinic Cohort Study of Oophorectomy and Aging examining the rela- 
tion between the bilateral and unilateral oophorectomy before the onset of natural menopause and the risk of cognitive impairment and dementia. This study showed that the risk increased with younger age at oophorectomy [8]. The increased risk was restricted to women who did not take oestrogen replacement therapy between the surgery and the age of 50 years. In this same study, women who underwent bilateral oophorectomy before 45 years of age experienced an increased mortality for neurological and mental disorders [8]. The authors explained also an effect of unilateral oophorectomy by the premature oestrogen deficiency caused by concurrent hysterectomy (70\% of all unilateral oophorectomies). Hysterectomy may compromise the blood supply to the remaining ovary, leading to premature ovarian failure. What was also reported was significantly earlier age at initiation of oestrogen treatment in the perimenopausal period for women who underwent unilateral oophorectomy with or without hysterectomy. These findings support the hypothesis that unilateral oophorectomy or concurrent hysterectomy may contribute to $\mathrm{POI}$ and that the association between oophorectomy and increased risk of cognitive impairment or dementia may be explained by a deficit in progesterone and testosterone. The association between oophorectomy and increased risk of cognitive impairment or dementia may be due to one or more susceptibility genes that increase the risk of both outcomes independently. Genetic variants may determine ovarian diseases prompting oophorectomy, or they may cause uterine diseases or symptoms that prompt hysterectomy, in turn, prompting oophorectomy.

Nappi et al. reported that women who underwent bilateral oophorectomy performed worse on certain memory tasks and that lower scores were corelated with younger age of oophorectomy [9]. Farrag et al. reported that women after bilateral oophorectomy had significantly decreased global cognitive scores and memory scores 3-6 months after surgical menopause in comparison with premenopausal controls and that lower performance scores corelated with lower oestradiol levels [10]. Sherwin compared cognitive functions before and after surgical menopause in 50 operated women (mean age 45.4 years) and reported a reduction in cognitive functions due to surgery if no hormone therapy was given [11]. The same author reported that hysterectomy with ovarian conservation and surgical menopause with subsequent menopausal replacement therapy caused no decline in cognitive function [11]. The negative effect of surgical menopause on memory and cognitive functions was not confirmed by studies of Kritz-Silverstein and Barrett-Connor [12] and Kok et al. [13].

The positive effect of oestrogen on cognitive functions was shown also in studies examining the impact of menopausal hormonal therapy on the risk of Alzheimer disease (AD) $[4,14,15]$. These studies reported a $20 \%$ to $40 \%$ reduction in risk of AD for women who used oestrogen treatment after menopause. The results of the Women's Health Initiative (WHI) clinical trials showed an increased risk of dementia in women treated by either oestrogen alone or oestrogen plus progestin after the age of 65 years [16]. These data suggest that oestrogen has a protective effect on the brain if given to women who underwent oophorectomy before reaching natural menopause, or if given in the perimenopausal and early postmenopausal years to women with natural menopause (critical window for neuroprotection). By contrast, it has a deleterious effect on the brain if started many years after the onset of natural menopause.

\section{Parkinsonism}

Both laboratory research and clinical studies suggest that there is an association between $\mathrm{POI}$ and an increased risk of Parkinsonism [3].

Laboratory studies reported several mechanisms of neuroprotective effects in animal models of Parkinson's disease (PD): oestrogen inhibited the neurotoxic action of neurotoxins: 1-methyl-4-phenyl-1,2,3,6-tetrahydropyridine, 6-hydroxydopamine, and methamphetamine; it reduced oxidative stress, upregulated neurotrophic factors, and protected dopaminergic neurons against apoptosis; oestrogen was protective of dopaminergic neurons in primate models [17].

Among the clinical studies, the Mayo Clinic Cohort Study of Oophorectomy and Aging showed an increased risk of Parkinsonism for women who underwent unilateral oophorectomy or bilateral oophorectomy [18]. The findings for unilateral oophorectomy were significant only when the oophorectomy was done before 42 years of age. Other studies give conflicting results. Benedetti et al. reported a link between surgical menopause and increased risk of PD, between early age at menopause and increased risk of PD, and between oestrogen treatment after menopause and decreased risk of PD [19]. Other reports did not confirm this [20, 21].

\section{Genetic diseases including premature ovarian insufficiency as one of the clinically rellevant features}

Among genetic diseases including $\mathrm{PO}$ as one of the clinically relevant features, the most important are ovarioleukodystrophy and fragile X-associated tremor/ ataxia syndrome (FXTAS).

\section{Ovarioleukodystrophy}

Ovarioleukodystrophy is an association of POI and vanishing white matter disease (VWM). VMN was de- 
scribed by Van der Knaap in 1996. This is a rare disease caused by a mutation in one of the subunits of eukaryotic initiation factor 2B (EIF2B). The symptoms combine progressive subcortical cognitive decline with predominant behavioural disorders and a progressive spastic paraparesis in association with symmetric cystic changes in both hemispheres of the brain [22]. For patients presenting with $\mathrm{POI}$ with neurological signs the MRI and screening of the EIF2B mutations is clinically indicated [23].

\section{Fragile X-associated tremor/ataxia syndrome}

FXTAS is a genetically-related disorder to fragile $X$ syndrome. Fragile $X$ syndrome is the second most common cause of intellectual disability (mental retardation) after Down's syndrome. Symptoms often include mild to moderate intellectual disability. Physical features may include a long and narrow face, large ears, flexible fingers, and large testicles. About a third of those affected have features of autism such as problems with social interactions and delayed speech. Hyperactivity is common and seizures occur in about $10 \%$ of patients. It is more frequent in males than in females.

FXTAS occurs typically after the age of 50 years, and its frequency increases with age. It is more frequent in males than in females [24]. Its clinical profile is variable and primarily characterised by tremor and ataxia. Other reported clinical characteristics include $\mathrm{POI}$, executive dysfunction, difficulty with social skills, Parkinsonism, polyneuropathy, thyroid dysfunction, depression, and anxiety [25].

Both fragile $X$ syndrome and FXTAS are related to the molecular defect of the FMR1 gene. Full mutation of the gene causes fragile $X$ syndrome, whereas a partial defect (premutation) causes FXTAS. FXTAS premutation is a CGG codon expansion ranging from 55 to 200 repetitions in the FMR1 gene located on the $\mathrm{X}$ chromosome [24].

FMR1 testing makes it possible to discover predisposition to $\mathrm{POI}$, and consequently to lower fertility or even cause infertility. The test result is especially important to women who are postponing the decision about having children. The test should be performed in the case of female patients with essential tremor and learning disabilities and autistic features in relatives [26].

\section{Conclusions}

POI may be related to severe climacteric symptoms, higher risk of dementia, cognitive function impairment, and Parkinsonism. Consequently, diagnosis of POI is an indication to menopausal replacement therapy. Conversely, some neurological diseases and signs, both in the clinical picture and in the family of the patient, may be related to higher risk of $\mathrm{POI}$ in the future.

\section{Disclosure}

The author reports no conflict of interest.

\section{References}

1. Schindler AE. Climacteric symptoms and hormones. Gynecol Endocrinol 2006; 22: 151-154.

2. Gibson-Helm M, Teede H, Vincent A. Symptoms, health behawior and understanding of menopause therapy in women with premature menopause. Climacteric 2014; 17: 666-673.

3. Vujović S, Ivović M, Tancić-Gajić M, et al. Premature ovarian failure. Srp Arh Celok Lek 2012; 140: 806-811.

4. Monk D, Brodaty H. Use of estrogens for the prevention and treatment of Alzheimer's disease. Dement Geriatr Cogn Disord 2000; 11: 1-10.

5. Arevalo MA, Azcoitia I, Garcia-Segura LM. The neuroprotective actions of oestradiol and oestrogen receptors. Nat Rev Neurosci 2015; 16: 17-29.

6. Lan YL, Zhao J, Li S. Update on the neuroprotective effect of estrogen receptor $\alpha$ against Alzheimer's disease. J Alzheimers Dis 2015; 43: 1137-1148.

7. Nofer JR. Estrogens and atherosclerosis: insights from animal models and cell systems. J Mol Endocrinol 2012; 48: R13-R29.

8. Rocca WA, Shuster LT, Grossardt BR, et al. Long-term effects of bilateral oophorectomy on brain aging: unanswered questions from the Mayo Clinic Cohort Study of Oophorectomy and Aging. Womens Health (Lond) 2009; 5: 39-48.

9. Nappi RE, Sinforiani E, Mauri M, et al. Memory functioning at menopause: impact of age in ovariectomized women. Gynecol Obstet Invest 1999; 47: 29-36.

10. Farrag AK, Khedr EM, Abdel-Aleem H, Rageh TA. Effect of surgical menopause on cognitive functions. Dement Geriatr Cogn Disord 2002; 13 : 193-198.

11. Sherwin BB. Estrogen and/or androgen replacement therapy and cognitive functioning in surgically menopausal women. Psychoneuroendocrinology 1988; 13: 345-357.

12. Kritz-Silverstein D, Barrett-Connor E. Hysterectomy, oophorectomy, and cognitive function in older women. J Am Geriatr Soc 2002; 50: 55-61.

13. Kok HS, Kuh D, Cooper R, et al. Cognitive function across the life course and the menopausal transition in a British birth cohort. Menopause 2006; 13: 19-27.

14. Yaffe K, Sawaya G, Lieberburg I, Grady D. Estrogen therapy in postmenopausal women: effects on cognitive function and dementia. JAMA 1998; 279: 688-695.

15. LeBlanc ES, Janowsky J, Chan BK, Nelson HD. Hormone replacement therapy and cognition: systematic review and meta-analysis. JAMA 2001; 285: 1489-1499.

16. Shumaker SA, Legault C, Kuller L, et al. Conjugated equine estrogens and incidence of probable dementia and mild cognitive impairment in postmenopausal women: Women's Health Initiative Memory Study. JAMA 2004; 291: 2947-2958.

17. Gillies GE, Murray HE, Dexter D, McArthur S. Sex dimorphisms in the neuroprotective effects of estrogen in an animal model of Parkinson's disease. Pharmacol Biochem Behav 2004; 78: 513-522.

18. Rocca WA, Bower JH, Maraganore DM, et al. Increased risk of parkinsonism in women who underwent oophorectomy before menopause. Neurology 2008; 70: 200-209.

19. Benedetti MD, Maraganore DM, Bower JH, et al. Hysterectomy, menopause, and estrogen use preceding Parkinson's disease: an exploratory case-control study. Mov Disord 2001; 16: 830-837.

20. Ragonese P, D'Amelio M, Salemi G, et al. Risk of Parkinson disease in women: effect of reproductive characteristics. Neurology 2004; 62: 2010-2014.

21. Ascherio A, Chen H, Schwarzschild MA, et al. Caffeine, postmenopausal estrogen, and risk of Parkinson's disease. Neurology 2003; 60: 790-795.

22. Herrera-García JD, Guillen-Martínez V, Creus-Fernández C, et al. Epilepsy and ovarian failure: Two cases of adolescent-onset ovarioleukodystrophy. Clin Neurol Neurosurg 2018; 165: 94-95.

23. Fogli A, Gauthier-Barichard F, Schiffmann R, et al. Screening for known mutations in EIF2B genes in a large panel of patients with premature ovarian failure. BMC Womens Health 2004; 4: 8. 
24. Rodriguez-Revenga L, Madrigal I, Pagonabarraga J, et al. Penetrance of FMR1 premutation associated pathologies in fragile $X$ syndrome families. Eur J Hum Genet 2009; 17: 1359e1362.

25. Merino S, Ibarluzea N, Maortua $\mathrm{H}$, et al. Associated clinical disorders diagnosed by medical specialists in 188 FMR1 premutation carriers found in the last 25 Years in the Spanish Basque Country: a retrospective study. Genes (Basel) 2016; 7: 90.

26. Lopez-Blanco R, Posada Rodríguez IJ, Benito-Leon J. Learning disability in a son and premature ovarian failure as clinical pointers to identify a premutation on the $\mathrm{X}$ chromosome in a female with long-standing tremor. Parkinsonism Relat Disord 2017; 42: 107e108. 